\title{
Alignment Efficiency of Open-Coil Spring Ver- sus Conventional Technique in Relieving Anterior Crowding: A Single-Center Randomized Clinical Trial
}

\author{
Amin Aminian ${ }^{1}$, Zahra Tavakolinejad* ${ }^{(D)}$, Hamed Ebrahimnejad, \\ Niloufar Azami ${ }^{4}$ \\ ${ }^{1}$ Department of Orthodontics, Kerman Dental School, Kerman University of Medical Sciences, Kerman, \\ Iran. \\ ${ }^{2}$ Department of Orthodontics, Rafsanjan Dental School, Rafsanjan University of Medical Sciences, Rafsan- \\ jan, Iran. \\ ${ }^{3}$ Oral and Dental Diseases Research Center, Kerman University of Medical Sciences, Kerman, Iran. \\ ${ }^{4}$ Private practice, Houston, TX.
}

\begin{tabular}{l} 
ARTICLE INFO \\
\hline Article History \\
Received:May 2021 \\
Accepted: Apr 2021 \\
ePublished: June 2021 \\
Corresponding author: \\
\hline Zahra Tavakolinejad, \\
Department of \\
Orthodontics, Rafsan- \\
jan Dental School, \\
Rafsanjan University \\
of Medical Scienc- \\
es, Rafsanjan, Iran. \\
Email: \\
Ortho.2017@yahoo. \\
com
\end{tabular}

\section{Introduction}

Improving the treatment efficiency is of particular importance in orthodontic treatment. Reduction of treatment duration, providing comfort, and achieving long-term stability are all based on treatment mechanics. ${ }^{(1,2)}$ Applying light and continuous forces will result in controlled tooth movement within the alveolar bone without any permanent damage. ${ }^{(3,4)}$ Tooth Movement

\begin{abstract}
Background and Aim: This study compares a new technique for teeth alignment to the conventional method. The teeth were aligned using an open-coil spring, i.e. simultaneous space opening and alignment, on round nickel-titanium (NiTi) archwires. The purpose of this 2-arm parallel trial (1:1 allocation ratio) was to evaluate the alignment efficiency of conventional and open-coil spring techniques in anterior crowding treatment.

Materials and Methods: In this clinical trial study, sixty-two non-extraction patients (70 dental arches: 24 upper arches and 46 lower arches) with $\geq 3 \mathrm{~mm}$ of Little's Irregularity Index (LII) were recruited in two groups based on the minimization method. Blinding was applicable for outcome assessment only. In one group, the teeth were aligned using an open-coil spring, whereas the alignment in the other group was done conventionally, (space opening on steel wires followed by alignment with an auxiliary NiTi wire). The alignment duration was the primary outcome that was investigated for survival analysis and alignment rate ratios, and levels of crowding were calculated with Cox proportional hazard regression. The pre- and post-treatment intercanine widths were also recorded as the secondary outcome. The chi-square and t-test were used to compare other variables between the groups. The level of statistical significance was set at $\mathrm{P}<0.05$.

Results: There was no significant difference in the alignment duration between the conventional (185.48 \pm 74.82 days) and open-coil (179.19 \pm 64.15 days) groups $(\mathrm{P}=0.725)$. No harm was detected using the mentioned methods.

Conclusion: The use of open-coil spring over brackets on NiTi or steel wires does not seem to affect anterior crowding treatment time.
\end{abstract}

J Res Dent Maxillofac Sci 2021;6(3):31-39.

Keywords: Alignment, Anterior Crowding, Randomized Clinical Trial,

Although biological factors are primarily beyond our control, an orthodontist can improve the efficiency of treatment through the choice of bracket system, type of archwire, and mechanotherapy. ${ }^{(5)}$

Anterior teeth crowding is often the chief complaint of orthodontic patients. ${ }^{(6,7)}$

\footnotetext{
Copyright (C) 2021 Faculty of Dentistry, Tehran Medical Sciences, Islamic Azad University.

This work is licensed under a Creative Commons Attribution-NonCommercial 4.0 International license (https://creativecommons.org/licenses/by$n c / 4.0 /)$. Noncommercial uses of the work are permitted, provided the original work is properly cited.
} 
In non-extraction cases, alignment of a crowded dental arch could be achieved by either increasing the arch length or decreasing the mesiodistal width of teeth through stripping.

Two techniques could be used to improve the arch length in a fixed orthodontic treatment: first, crimping a stop on the archwire mesial to the molar tube to keep the archwire in an advanced position relative to anterior bracket slots, and second, by using active coil springs on an archwire to create space for crowded teeth. The applied force from the coil spring should be light with no deformation in the archwire. Both methods would result in the proclination of the incisors. ${ }^{(8,9)}$

Open-coil springs apply a bilateral pushing force and have various applications in orthodontics. ${ }^{(10)}$ In contrast to the conventional alignment technique, open-coil springs can be used on nickel-titanium (NiTi) archwires from the first steps of treatment with the simultaneous engagement of the archwire into the severely misaligned teeth brackets. ${ }^{(11,12)}$ However, their efficiency compared to the conventional method of alignment has not been investigated.

This clinical trial aimed to compare the efficiency of the conventional technique in the alignment of anterior crowding versus the open-coil spring approach. Also, changes in the intercanine width, as an index of long-term stability, were evaluated in the two groups.

\section{Materials and Methods}

The Ethics Committee of KMU.REC.1394.2 granted ethical approval for this clinical trial. This was a 2-arm parallel (1:1 allocation ratio) randomized controlled clinical trial. All the participants were given oral and written explanations, and informed consent was obtained from all patients and the parents of adolescent patients. The data are presented according to the CONSORT statement (Figure 1). ${ }^{(13)}$

Patients were recruited from the department of orthodontics between October 2016 and June 2017 based on the following inclusion criteria: age from 11 to 36 years at the start of treatment, Little's Irregularity Index $\geq 3 \mathrm{~mm}$, non-extraction orthodontic treatment plan, fully erupted permanent teeth anterior to the first molars, and healthy periodontal tissues. ${ }^{(14-17)}$ Patients with any spacing in the anterior segment, missing, impacted or supernumerary teeth anterior to the first molars, high buccal canines, dilacerated roots, crowding in the posterior teeth, posterior cross-bite, systemic medical problems, previous active orthodontic treatment, clefts and other craniofacial anomalies and those who were taking medications that affect bone metabolism were excluded from the study. The patient was also banned if any bracket was debonded more than once during treatment. ${ }^{(14,18,19)}$ The demographic and clinical characteristics of the patients are shown in Table 1. The two study groups were matched according to sex, age, malocclusion type, and the amount of crowding, and no significant difference was noticed (Table 1).

Metal $0.022 \times 0.028$ " MBT prescription brackets (Sprint series, Forestadent, Germany) were bonded directly for all patients using light-cured composite resin (Light Bond, USA). The bands were cemented on the first molars with glass ionomer.

In the conventional technique, alignment archwires of $0.012 ", 0.014 ", 0.016 "$, and 0.018 " NiTi (TruFlexTM, OrthoTechnology Inc., USA) were used sequentially depending on the amount of crowding based on the clinician's decision without engaging the lingually-positioned teeth until the partial alignment was achieved. Then, a NiTi open-coil spring (OrthoTechnology Inc., USA) was used on a 0.016 " or 0.018 " stainless steel archwire (TruForceTM, OrthoTechnology Inc., USA) to create space, and finally, when enough space was gained, a 0.012 " superelastic A-NiTi archwire was utilized as an auxiliary wire to move the misaligned tooth into the arch..$^{(9)}$

In the open-coil spring approach, a NiTi opencoil spring (0.010"* 0.030 ") was used from the first appointment on 0.012" A-NiTi archwires. The archwire was engaged on all teeth (even the lingually-positioned teeth) by elastomeric or stainless steel ligatures unless severe overlap of teeth made it impossible. In the latter cases, the bracket was bonded and engaged as soon as enough access to the labial surface of the aforementioned teeth was created; the process was continued with other archwires (0.014", 0.016", 0.018 " NiTi and 0.016 " or 0.018 " stainless steel) until the alignment of teeth was achieved. The archwire was cut at the distal of molar bands and was not cinched (Figure 2). 


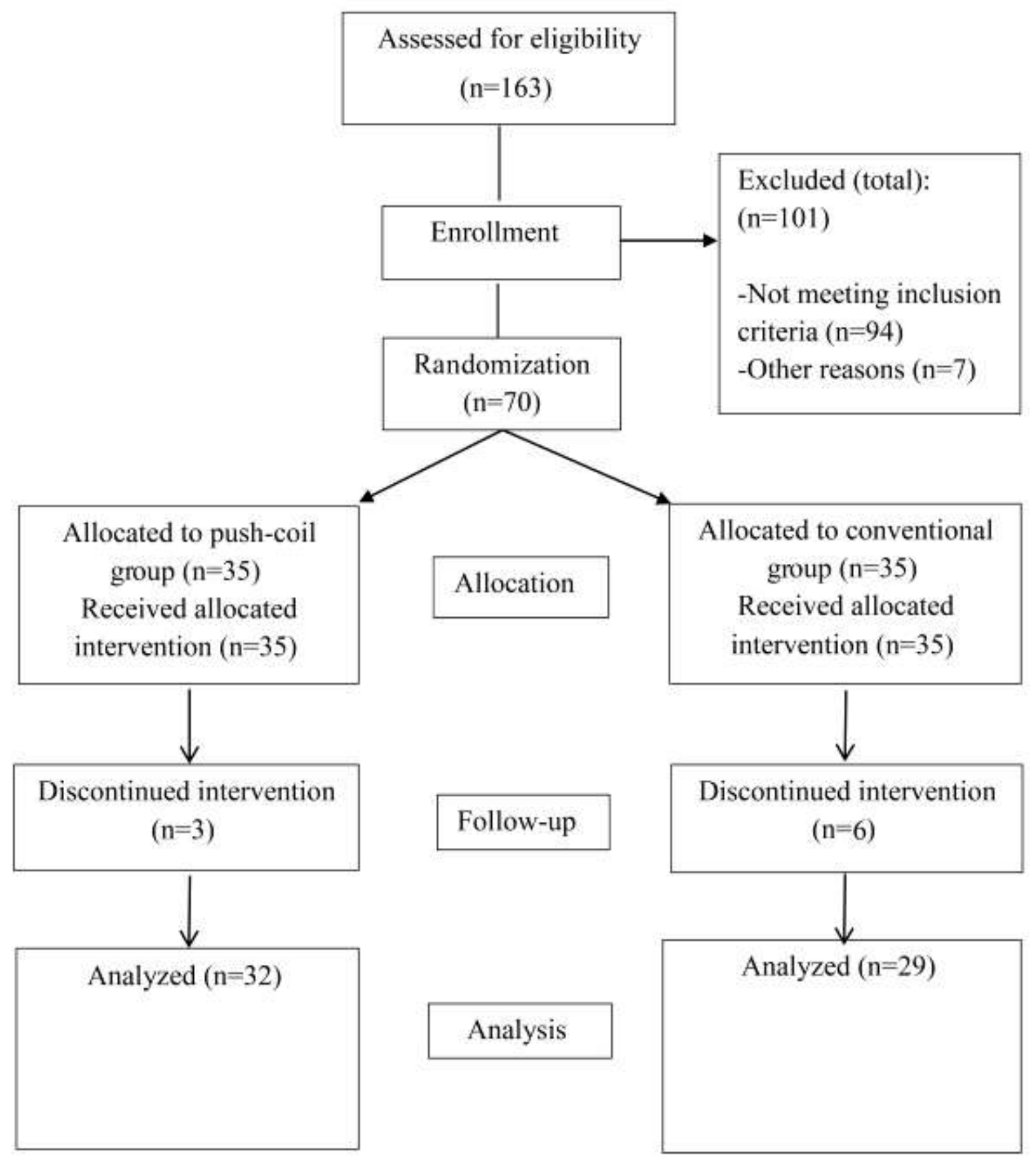

Figure 1. CONSORT flowchart diagram of the study 
Table 1. Patients' demographic and clinical characteristics

\begin{tabular}{|c|c|c|c|c|c|c|c|}
\hline & $\begin{array}{c}\text { Total } \\
(\mathrm{n}=61) \\
\text { mean or } \%\end{array}$ & SD & $\begin{array}{l}\text { Open-coil } \\
\text { spring } \\
(\mathrm{n}=32) \\
\text { mean or } \%\end{array}$ & SD & $\begin{array}{c}\text { Conventional } \\
(\mathrm{n}=29) \\
\text { mean or } \%\end{array}$ & $\mathrm{SD}$ & P-value* \\
\hline \multicolumn{8}{|c|}{ Demographic Characteristics } \\
\hline Age (year) & 18.74 & 6.46 & 18.38 & 5.74 & 19.14 & 7.26 & 0.649 \\
\hline \multicolumn{8}{|l|}{$\operatorname{Sex}(\%)$} \\
\hline Male & 24.6 & & 21.9 & & 27.6 & & \multirow{2}{*}{0.605} \\
\hline Female & 75.4 & & 78.1 & & 72.4 & & \\
\hline \multicolumn{8}{|c|}{ Clinical Characteristics } \\
\hline \multicolumn{8}{|c|}{ Angle class (\%) } \\
\hline I & 57.4 & & 62.5 & & 51.8 & & \multirow{3}{*}{0.298} \\
\hline II & 26.2 & & 28.1 & & 24.1 & & \\
\hline III & 16.4 & & 9.4 & & 24.1 & & \\
\hline \multicolumn{8}{|l|}{$\operatorname{Arch}(\%)$} \\
\hline Upper & 32.8 & & 37.5 & & 27.6 & & \multirow{2}{*}{0.410} \\
\hline Lower & 67.2 & & 62.5 & & 72.4 & & \\
\hline $\begin{array}{l}\text { ATSALD } \\
(\mathrm{mm})\end{array}$ & 4.58 & 1.22 & 4.78 & 1.31 & 4.36 & 1.08 & 0.182 \\
\hline LII (mm) & 8.65 & 3.07 & 9.21 & 2.92 & 8.04 & 3.16 & 0.138 \\
\hline
\end{tabular}

NS, Not significant; ATSALD, Anterior tooth-size arch-length discrepancy; LII, Little's Irregularity Index; SD, standard deviation .P-value for comparison of group means by t-test or differences in proportions by chi-square and Fisher>s exact test*

During the study, no inter-arch elastics, expansion appliances, headgear or any other extraoral or intraoral devices were used. Moreover, no interproximal reduction was made before the completion of the alignment phase..$^{(14-17)}$

Before the orthodontic treatment, the anterior crowding was measured by Little's irregularity index (LII) and anterior tooth-size arch-length discrepancy (ATSALD). ${ }^{(9,20-22)}$ LII, which is the sum of the linear horizontal displacement of the five contact points from the mesial aspect of the canine to the mesial aspect of the contralateral canine, was measured using a fine-tip digital caliper. The ATSALD was measured as the difference between the arch length and the sum of the mesiodistal widths of the six anterior teeth. The arch length was measured from the distal contact of the canine to the distal contact of the canine on the opposite side.

The initial intercanine width (ICW1) was measured on dental casts using a digital caliper (precision of $0.01 \mathrm{~mm}$ ). Two orthodontists carried out all the measurements, and the mean was recorded.The methods to assess the reliability measurement, the ATSALD and LII measurements, were repeated on 20 randomly selected dental casts after one month.
The t-test showed no significant difference between the two measurements $(\mathrm{P}=0.214)$.

The bonding date was recorded as the start of treatment (T1). All patients were followed every four weeks until the two orthodontists confirmed the complete correction of anterior teeth crowding (canine to canine) based on the occlusal photographic images and clinical judgments. If no rotation or displacement was observed in the contact points of the anterior teeth and the 0.018 " stainless steel archwire was engaged fully, the alignment process was considered done, and the date was recorded as the end of the alignment phase (T2). The alignment duration (T2-T1) was recorded for each patient as the primary outcome. The alignment rate was calculated to evaluate the efficiency of the two methods. Once the alignment phase was completed, the changes in the intercanine width (ICW2-ICW1) were measured as the secondary outcome using digital calipers (precision of $0.01 \mathrm{~mm}$ ) directly on the dental arch of the patients.

A sample size of 25 dental arches in each group was calculated based on power analysis (power $=80 \%, \alpha=0.05$ ). However, considering the dropout rate, 35 dental arches were recruited in each group. 
Patients were randomly allocated to the conventional or open-coil spring groups based on the minimization (covariate adaptive randomization) method using the Minim software (Plymouth, UK).

Blinding of clinician was not possible, but the statistician was blind to the assigned treatment. Random allocation was confirmed by the similarities in the demographics of each group

(Table 1).

Statistical analysis

The normality of data was assessed by the Kolmogorov-Smirnov test. The analysis of variance (ANOVA) was used to study the difference between the rates of treatment in various types of malocclusion. The chi-square and t-test were used to compare other variables between the groups. The time needed for alignment was investigated with survival analysis, and alignment rate ratios for the type of method and crowding were assessed with the Cox proportional hazard regression. The level of statistical significance was set at $\mathrm{P}<0.05$ with a $95 \%$ confidence interval (CI). All analyses were conducted with SPSS software (version 22.0, SPSS Inc., Chicago, IL, USA).

\section{Results}

Sixty-two patients (70 dental arches) were randomized in a 1:1 ratio to either open-coil spring or conventional groups; however, 9 dental arches dropped out during the follow-up sessions (Figure 1). Patient recruitment commenced in October 2016 and ended in June 2017.In the open-coil group, 3 arches were excluded from the study. Two patients were excluded due to repeated bracket debonding and one patient due to poor cooperation in regular visits. Finally, 32 dental arches were analyzed in this group.In the conventional group, 6 arches were excluded from the study: two due to repeated bracket debonding and four due to poor cooperation in regular visits. Finally, 29 dental arches were analyzed in this group.Table 1 shows the demographic and clinical characteristics of the two groups including age, gender, Angle classification, dental arch (upper or lower), crowding, and LII. No significant difference was noted in these variables between the two groups, which guaranteed the random allocation.Among the fifty-four patients (61 dental arches), whose outcomes were analyzed, there was no significant difference in the alignment duration between the conventional (185.48 \pm 74.82 days) and open-coil (179.19 \pm 64.15 days) groups $(\mathrm{P}=0.725)$. Table 2 shows the alignment rate ratios based on the applied approach and various types of crowding. Generally, according to the overcrowding (TSALD), the Cox proportional hazard regression revealed that the open-coil spring had a 1.49 times higher probability of earlier crowding correction, which was not statistically significant $(\mathrm{P}=0.150)$. On the other hand, based on the LII, the hazard ratio of the open-coil group was 2.15, which was significant $(\mathrm{P}=0.011)$. Severe crowding cases showed statistical significance with a hazard ratio of 0.30 and 0.20 in the open-coil and conventional groups, respectively. Severe LII arches had a significant hazard ratio of 0.38 in the conventional group $(\mathrm{P}=0.030)$. The open-coil technique showed a significant hazard ratio in severe LII cases ( $\mathrm{P}=0.021$; Table 2).

Figure 3 depicts the Kaplan-Meier survival curves for the two groups based on the required alignment time.The changes in the intercanine width were smaller in the open-coil group, but the difference was not statistically significant $(\mathrm{P}=0.681)$. The patients' gender and type of malocclusion did not have any significant impact on the alignment rate. However, in the open-coil group, the rate of alignment in the upper arch was significantly higher than that of the lower arch $(\mathrm{P}=0.013)$.
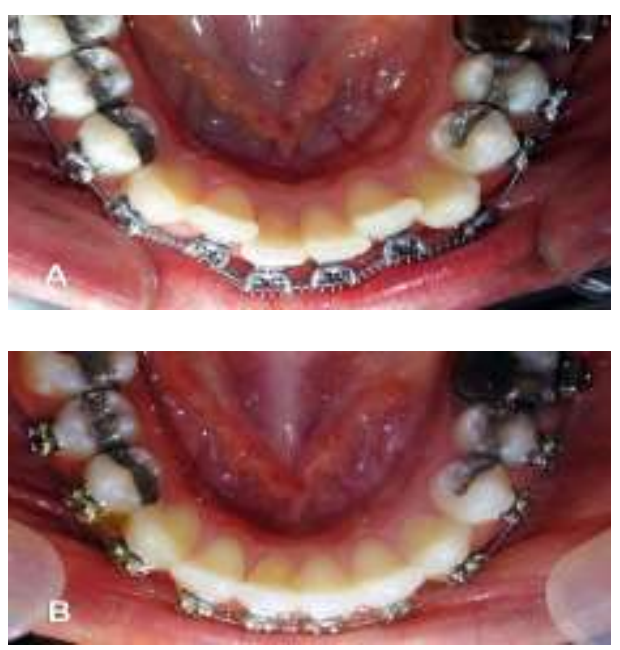

Figure 2. Using the push-coil spring. Before (A) and after (B) alignment. 
Table 2. Alignment rate ratios obtained from Cox proportional hazards regression

\begin{tabular}{|c|c|c|c|}
\hline & $\begin{array}{c}\text { Adjusted hazard } \\
\text { ratio } \dagger\end{array}$ & $\begin{array}{c}95 \% \text { Confidence } \\
\text { Interval }\end{array}$ & P-value \\
\hline $\begin{array}{l}\text { Based on TSALD } \\
\text { Conventional } \\
\text { Open-coil }\end{array}$ & $\begin{array}{c}\text { Baseline } \\
1.49\end{array}$ & $0.87-2.56$ & 0.150 \\
\hline $\begin{array}{l}\text { Based on LII } \\
\text { Conventional } \\
\text { Open-coil }\end{array}$ & $\begin{array}{c}\text { Baseline } \\
2.15\end{array}$ & $1.20-3.87$ & $0.011 *$ \\
\hline $\begin{array}{l}\text { Open-coil group } \\
\text { Moderate TSALD }(\leq 4 \mathrm{~mm}) \\
\text { Severe TSALD }(>4 \mathrm{~mm})\end{array}$ & $\begin{array}{c}\text { Baseline } \\
0.30\end{array}$ & $0.13-0.68$ & $0.004 *$ \\
\hline $\begin{array}{l}\text { Conventional group } \\
\text { Moderate TSALD }(\leq 4 \mathrm{~mm}) \\
\text { Severe TSALD }(>4 \mathrm{~mm})\end{array}$ & $\begin{array}{c}\text { Baseline } \\
0.20\end{array}$ & $0.09-0.48$ & $0.000 *$ \\
\hline $\begin{array}{l}\text { Open-coil group } \\
\text { Moderate LII }(\leq 7 \mathrm{~mm}) \\
\text { Severe LII }(>7 \mathrm{~mm})\end{array}$ & $\begin{array}{c}\text { Baseline } \\
0.88\end{array}$ & $0.37-2.11$ & 0.777 \\
\hline $\begin{array}{l}\text { Conventional group } \\
\text { Moderate LII }(\leq 7 \mathrm{~mm}) \\
\text { Severe LII }(>7 \mathrm{~mm})\end{array}$ & $\begin{array}{c}\text { Baseline } \\
0.38\end{array}$ & $0.16-0.91$ & $0.030 *$ \\
\hline $\begin{array}{l}\text { Moderate TSALD }(\leq 4 \mathrm{~mm}) \\
\text { Conventional } \\
\text { Open-coil }\end{array}$ & $\begin{array}{c}\text { Baseline } \\
1.99\end{array}$ & $0.84-4.74$ & 0.120 \\
\hline $\begin{array}{l}\text { Severe TSALD }(>4 \mathrm{~mm}) \\
\text { Conventional } \\
\text { Open-coil }\end{array}$ & $\begin{array}{c}\text { Baseline } \\
1.44\end{array}$ & $0.60-3.42$ & 0.415 \\
\hline $\begin{array}{l}\text { Moderate LII }(\leq 7 \mathrm{~mm}) \\
\text { Conventional } \\
\text { Open-coil }\end{array}$ & $\begin{array}{c}\text { Baseline } \\
0.89\end{array}$ & $0.32-2.47$ & 0.825 \\
\hline $\begin{array}{l}\text { Severe LII }(>7 \mathrm{~mm}) \\
\text { Conventional } \\
\text { Open-coil }\end{array}$ & $\begin{array}{c}\text { Baseline } \\
2.41\end{array}$ & $1.14-5.09$ & $0.021 *$ \\
\hline
\end{tabular}

ATSALD, Anterior tooth-size arch-length discrepancy; LII, Little's Irregularity Index .Hazard ratios adjusted for demographic characteristics and Angle class $\uparrow$ .Significant*

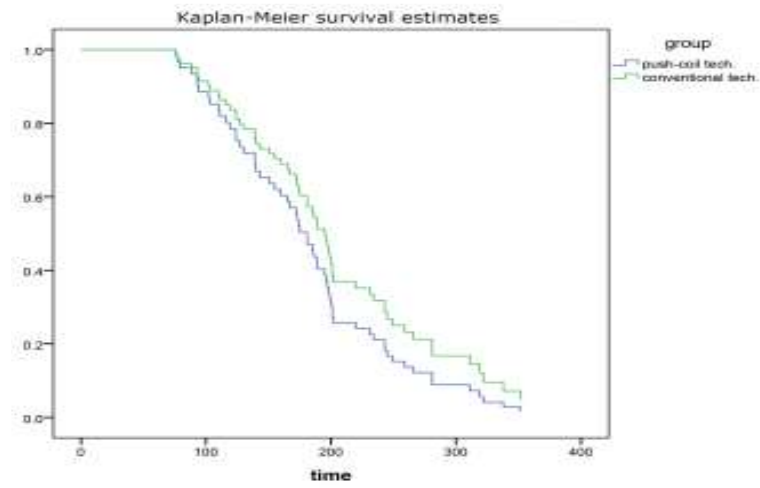

Figure 3. Kaplan-Meier survival curves for the two groups based on alignment time. Lack of separation implies no significant effect on treatment duration 


\section{Discussion}

This study is the first clinical trial that compares the alignment efficiency of this particular open coil spring application on NiTi wires with the aforementioned conventional method.

NiTi open coil springs and superelastic NiTi wires generate light and almost constant forces over a wide range of activation. ${ }^{(9,23-25)}$ The combination of these two elements could be used in the initial alignment phase of orthodontic treatments that require space, long-range of action, and constant light forces. ${ }^{(9,23)}$ However, light NiTi wires will be deformed by the pushing force that NiTi coil springs exert unless the NiTi coil is activated slightly. ${ }^{(9)}$

Therefore, in this study, to decrease the amount of undesirable wire deformation, especially at the beginning stages of the alignment, misaligned teeth were engaged to the archwire from the very beginning of the alignment phase. This would cause simultaneous space opening and alleviation of anterior crowding similar to the application of crimpable stops on NiTi wires; ${ }^{(9)}$ however, in this approach, space opening mechanics would be more localized and would just target the crowded segment of the arch.

No apparent arch-form distortion, inappropriate tipping, and rotation of non-crowded teeth were noticed during the alignment phase, which could be due to the neutralizing moments and forces of wire engagement to lingualized teeth, which can almost balance their space opening counterparts even though this system is indeterminable. The magnitude of these complicated groups of counterbalancing moments and forces cannot be predicted precisely. This, in turn, allowed us to activate the open-coil spring on preliminary archwires more than the conventional approaches with round NiTi wires. Furthermore, to make the conditions closer to the clinical situations, the wires were changed based on the clinical judgment of the clinician.

This study showed that in the NiTi coil spring group, using TSALD and LII, alignment was 1.49 and 2.15 times faster than the conventional method, respectively. Moreover, based on the results obtained by LII, the difference between the alignment rates in the two groups was statistically significant. In the present study, the open-coil approach showed better clinical effects when LII was applied. LII and TSALD represent different aspects of discrepancy where LII measures the contact displacement and TSALD is the amount of space deficiency in the arch. Bypassing the lingual teeth in the conventional method can sometimes cause more contact displacement. However, in the NiTi coil spring method, ligating all teeth from the beginning leads to the gradual correction of LII.

The average time required to complete the alignment in this newer method is 6.29 days less than the conventional method with no clinical significance. However, the patient's satisfaction might be higher since teeth appearance is taken care of and improved from the very beginning, unlike the conventional approach in which severely displaced teeth usually would be aligned by piggyback wires after reaching stiff archwires later in the alignment phase. ${ }^{(9)}$

In cases in which one or two teeth are positioned out of the ideal arch form, and there is not enough space, space opening with the conventional approach is usually straightforward, but as the number of misaligned teeth increases, problems such as wire traveling and soft tissue irritation increase as well. ${ }^{(9)}$ Also, in such conditions, the probability of specific side effects, such as the labial movement of non-bypassed teeth increases, which can cause a gingival recession and bite interferences, although stiff archwires would control unwanted tipping and rotational movements of the anchored teeth. In this new method, these problems have been reduced as more teeth are engaged into the archwire from the beginning of the alignment, and therefore, space-opening forces are not concentrated on the most protruded teeth. One can make better sense of the alignment mechanism when intercanine width and incisors' inclination are compared before and right after treatment. As this study was conducted in the alignment orthodontic treatment phase (the orthodontic treatment was not complete), no cephalometric records were obtained due to ethical considerations. In the push-coil group, the patients did not express any additional discomfort probably due to the engagement of the push coil on the brackets. It is recommended to compare the attitude and comfort (pain experience) of patients in this method to that of the conventional method. 
It is also suggested to compare treatment duration and arch length and width changes with that of other techniques, such as self-ligating systems, which claim to have improved results.

With improved mechanotherapy skills and a better case selection, one could likely achieve better results with this new method in terms of the alignment rate. Furthermore, this approach could be used in any case that requires simultaneous space opening and alignment, especially where proclination of the incisors is beneficial in the alignment phase as in Class II division 2 cases that require growth modification or decompensation for orthognathic surgery.

\section{Conclusion}

The results of this study showed that there was no significant difference in the alignment duration between the two methods. As expected, the amount of crowding, regardless of the applied method, has a significant impact on the rate of teeth alignment.

\section{Acknowledgments}

The authors would like to extend their sincere appreciation to Davood Abrishami for his useful help and support.

\section{References}

1. Lo Giudice A, Isola G, Rustico L, Ronsivalle V, Portelli M, Nucera R. The Efficacy of Retention Appliances after Fixed Orthodontic Treatment: A Systematic Review and Meta-Analysis. Appl Sci. 2020;10(9):3107.

2. Chow L, Goonewardene MS, Cook R, Firth MJ. Adult orthodontic retreatment: A survey of patient profiles and original treatment failings. Am J Orthod Dentofacial Orthop. 2020; 158(3):371-382.

3. Malek NH, Hasan H, Dasor MM. Partial least squares (pls) latent variable modelling approach for measuring duration of orthodontic treatment. Malays J Comput Sci. 2020;5(1):46979.

4. Yadav S, Markiewicz MR, Allareddy V. Dentoalveolar Distraction Osteogenesis for Rapid Maxillary Canine Retraction: An Overview of Technique, Treatment, and Outcomes. Oral Maxillofac Surg Clin. 2020;1;32(1):83-8.

5. Scott P, DiBiase AT, Sherriff M, Cobourne MT. Alignment efficiency of Damon3 self-ligating and conventional orthodontic bracket systems: a randomized clinical trial. Am J Orthod Dentofacial Orthop. 2008;134(4):470.e1-8.

6. Bernabé E, Flores-Mir C. Estimating arch length discrepancy through Little's Irregularity Index for epidemiological use. Eur J Orthod. 2006;28:269-73.

7. Eslambolchi S, Woodside DG, Rossouw PE. A descriptive study of mandibular incisor alignment in untreated subjects. Am J Orthod Dentofacial Orthop. 2008;133:343-53.

8. Fleming PS, DiBiase AT, Sarri G, Lee RT. Comparison of mandibular arch changes during alignment and leveling with
2 preadjusted edgewise appliances. Am J Orthod Dentofacial Orthop. 2009;136:340-7.

9. Proffit WR, Fields HW, Sarver DM. Contemporary orthodontics. 5th ed. Missouri: Mosby; 2013.

10. Brauchli LM, Senn C, Ball J, Wichelhaus A. Force levels of 23 nickel-titanium open-coil springs in compression testing. Am J Orthod Dentofacial Orthop. 2011;139:601-5. 11. Harradine N. Self-ligating brackets: theory, practice, and evidence. In: Graber LW, Vanarsdall RL, Vig KWL. Orthodontics: current principles \& techniques. 5th ed. Missouri: Mosby, 2012:607-11.

12. Bourke A, Daskalogiannakis J, Tompson B, Watson P. Force characteristics of nickel-titanium open-coil springs. Am J Orthod Dentofacial Orthop. 2010 Aug 1;138(2):142e1.

13. Moher D, Hopewell S, Schulz KF, Montori V, Gøtzsche PC, Devereaux PJ, et al. CONSORT 2010 explanation and elaboration: updated guidelines for reporting parallel group randomised trials. BMJ. 2010;340:c869.

14. Sebastian B. Alignment efficiency of superelastic coaxial nickel-titanium vs superelastic single-stranded nickel-titanium in relieving mandibular anterior crowding: A randomized controlled prospective study. Angle Orthod. 2012;82:703-8.

15. Pandis N, Polychronopoulou A, Katsaros C, Eliades T. Comparative assessment of conventional and self-ligating appliances on the effect of mandibular intermolar distance in adolescent nonextraction patients: a single-center randomized controlled trial. Am J Orthod Dentofacial Orthop. 2011;140:e99-e105.

16. Sandhu SS, Sandhu J. A randomized clinical trial investigating pain associated with superelastic nickel-titanium and multistranded stainless steel archwires during the initial leveling and aligning phase of orthodontic treatment. J Orthod. 2013;40:276-85.

17. Pandis N, Polychronopoulou A, Eliades T. Active or passive self-ligating brackets? A randomized controlled trial of comparative efficiency in resolving maxillary anterior crowding in adolescents. Am J Orthod Dentofacial Orthop. 2010;137:12.e1-6.

18. Ong E, Ho C, Miles P. Alignment efficiency and discomfort of three orthodontic archwire sequences: a randomized clinical trial. J Orthod. 2011;38:32-9.

19. Wahab RM, Idris H, Yacob H, Ariffin SH. Comparison of self- and conventional-ligating brackets in the alignment stage. Eur J Orthod. 2012;34:176-81.

20. Little RM. The irregularity index: a quantitative score of mandibular anterior alignment. Am J Orthod. 1975;68:55463.

21. Quaglio CL, Freitas KM, Freitas MR, Janson G, Henriques JF. Stability of maxillary anterior crowding treatment. Dental Press J Orthod. 2012;17:57-64.

22. Destang DL, Kerr WJ. Maxillary retention: is longer better? Eur J Orthod. 2003;25:65-9.

23. Bourke A, Daskalogiannakis J, Tompson B, Watson P. 24. Steinbach G, Armstrong D, Kharbanda OP, Petocz P, Darendeliler MA. An investigation of the forces used by clinicians to open spaces with coil springs. Aust Orthod. J 2006;22:115-20.

25. West AE, Jones ML, Newcombe RG. Multiflex versus superelastic: a randomized clinical trial of the tooth alignment ability of initial arch wires. Am J Orthod Dentofacial 
Cite this paper as: Aminian A, Tavakolinejad Z, Ebrahimnejad H, Azami N. Alignment Effi-

ciency of Open-Coil Spring Versus Conventional Technique in Relieving Anterior Crowding: A

Single-Center Randomized Clinical Trial. J Res

Dent Maxillofac Sci. 2021; 6 (3):31-39. 